\title{
Rooted trees for 3D Navier-Stokes equation
}

\author{
Massimiliano Gubinelli \\ Communicated by Charles Li, received November 22, 2005.
}

\begin{abstract}
We establish a representation of a class of solutions of 3d NavierStokes equations in $\mathbb{R}^{3}$ using sums over rooted trees. We study the convergence properties of this series recovering in a simplified manner some results obtained recently by Sinai and other known results for solutions in spaces of pseudo-measures introduced initially by Le Jan and Sznitman. The series representation make sense also in the critical case where there exists global solutions for small initial data and it allows the study of their long-time or small-distance behavior.
\end{abstract}

\section{Contents}

1. Introduction 161

2. Trees 163

3. Series representation 163

$\begin{array}{ll}\text { acknowledgment } & 168\end{array}$

$\begin{array}{ll}\text { References } & 169\end{array}$

Appendix A. Remarks on the spaces $\Phi(\alpha, \omega) \quad 169$

$\begin{array}{ll}\text { Appendix B. Proofs } & 170\end{array}$

\section{Introduction}

We consider the NS equation in the form

$$
v_{t}(k)=e^{-|k|^{2} t} v_{0}(k)+i \int_{0}^{t} e^{-|k|^{2}(t-s)} \int_{\mathbb{R}^{3}} d k^{\prime}\left\langle k, v_{s}\left(k-k^{\prime}\right)\right\rangle P_{k} v_{s}\left(k^{\prime}\right) d s
$$

where $v_{t} \in C\left(\mathbb{R}^{3} ; \mathbb{C}^{3}\right)$ is the Fourier transform of the velocity field, $\langle\cdot, \cdot\rangle$ is the scalar product in $\mathbb{C}^{3}$ and $P_{k}: \mathbb{C}^{3} \rightarrow \mathbb{C}^{3}$ is the projection on the directions orthogonal to

1991 Mathematics Subject Classification. 35, 76.

Key words and phrases. Navier-Stokes equations, rooted tree, pseudo-measure.

(C)2006 International Press 
the vector $k \in \mathbb{R}^{3}$, i.e. $P_{k} a=a-\langle k, a\rangle k|k|^{-1}$. Eq. (1) will be studied in the spaces $\Phi(\alpha, \alpha), \alpha \in[2,3)$ and where $v \in \Phi(\alpha, \alpha)$ if $v \in C\left(\mathbb{R}^{3} ; \mathbb{C}^{3}\right)$ with $k \cdot v(k)=0$ and

$$
\|v\|_{\alpha}=\sup _{k \in \mathbb{R}^{3}}|k|^{\alpha}|v(k)|<\infty .
$$

We will write $\alpha=2+\varepsilon$ with $\varepsilon \in[0,1)$.

The spaces $\Phi(\alpha, \alpha)$ are interesting because, in general, they contain solutions with infinite energy and enstrophy so classical results about existence and uniqueness of solutions do not apply.

In a series of papers, Sinai $[\mathbf{8}, \mathbf{9}, \mathbf{1 0}]$, studies eq. (1) in these spaces giving elementary proofs that in $\Phi(\alpha, \alpha)$ with $\alpha>2$, there is existence of unique local solutions and that these solutions survive for arbitrary large time if the initial condition is small enough.

Moreover in the "critical" space $\Phi(2,2)$ there is existence and uniqueness of global solutions for small initial data. This latter global result was initially proven by Le Jan and Sznitman [11] using a probabilistic representation (under the name of stochastic cascades) and afterwards reproved by Cannone and Planchon [5] in a more standard functional-analytic fashion.

The analysis of the equation (1) in various function spaces similar to $\Phi(\alpha, \alpha)$ is summarized in the work of Bhattacharya et al. in [1]. A more recent review of the current status of the stochastic cascades approach has been recently given by Waymire in $[\mathbf{1 2}]$.

We are interested in explicit series representations for these (local or global) solutions. When $\alpha>2$ Sinai $[\mathbf{9}]$ proved that the local solution can be represented by a absolutely convergent series and in $[\mathbf{1 0}]$ he analyzed this series with the aim of understanding better the growth of the various terms. A different series representation appears also in the book of Gallavotti [7].

Our contribution will be to prove yet another series representation for the solutions in $\Phi(\alpha, \alpha)$ including the critical case $\alpha=2$ which was left open by the analysis of Sinai. This series representation is indexed by rooted trees.

Rooted trees appear naturally in the series expansion of solutions to ODEs. They possess remarkable algebraic properties which were masterfully exploited by Butcher [4] to provide a general theory of Runge-Kutta (R-K) methods for numerical integration. Afterwards rooted trees appeared also in the work of Connes and Kreimer [6] on the mathematical structure of renormalization in quantum field theory. The work of Brouder $[\mathbf{2}, \mathbf{3}]$ gives a short overview of the algebraic properties of rooted trees and explore some connections between R-K methods and renormalization.

These widespread applications of rooted trees were the initial motivations for this work. In the following we show that rooted trees are a natural language in which the known results (and some new ones) about the eq. (1) in the spaces $\Phi(\alpha, \alpha)$ can be proven rather easily. Moreover the representation with a series indexed by rooted-trees can be controlled in a straightforward way and provide informations on the solutions themselves, like the behavior for large times or for large wave-vectors.

The plan of the paper is as follows. In Sec. 2 we introduce rooted trees which will be used in Sec. 3 to prove the series representation for the solutions of NS equation. Next in Sec. 3.1 we make some observations on the different nature of 
some classes of terms which contribute to the series and which appeared originally in the work of Sinai [10]. In App. A we review briefly, for sake of completeness, the question of existence and uniqueness problem for the equation (1) in the spaces $\Phi(\alpha, \omega)$. At the end, App. B collects some proofs.

\section{Trees}

A rooted tree is a graph with a special vertex called root such that there is a unique path from the root to any other vertex of the tree. Here some examples of rooted trees:

\section{. $: \forall \forall \forall$}

We draw the root at the bottom with the tree growing upwards (as real trees). In a rooted tree the order of the branches at any vertex is ignored so the following two are representations of the same tree:

\section{i.}

Given $k$ rooted trees $\tau_{1}, \cdots, \tau_{k}$ we define $\tau=\left[\tau_{1}, \cdots, \tau_{k}\right]$ as the tree obtained by attaching the $k$ roots of $\tau_{1}, \cdots, \tau_{k}$ to a new vertex which will be the root of $\tau$. Any tree can be constructed using the simple tree $\bullet$ and the operation $[\cdots]$, e.g.

$$
[\bullet]=\mathfrak{:} \quad[\bullet,[\bullet]]=\mathfrak{q}, \quad \text { etc. } .
$$

On trees we will define various functions. Denote $\mathcal{T}$ the set of all rooted trees and let $|\cdot|: \mathcal{T} \rightarrow \mathbb{N}$ the map which counts the number of vertices of the tree and which can be defined recursively as

$$
|\bullet|=1, \quad\left|\left[\tau_{1}, \ldots, \tau_{k}\right]\right|=1+\left|\tau_{1}\right|+\cdots+\left|\tau_{k}\right|
$$

moreover we define the tree factorial $\gamma: \mathcal{T} \rightarrow \mathbb{N}$ as

$$
\gamma(\bullet)=1, \quad \gamma\left(\left[\tau_{1}, \ldots, \tau_{k}\right]\right)=\left|\left[\tau_{1}, \ldots, \tau_{k}\right]\right| \gamma\left(\tau_{1}\right) \cdots \gamma\left(\tau_{k}\right) .
$$

Last, we define the symmetry factor $\sigma: \mathcal{T} \rightarrow \mathbb{N}$ : this is defined recursively as $\sigma(\bullet)=1$ and $\sigma\left(\left[\tau_{1} \cdots \tau_{n}\right]\right)=s\left(\tau_{1}, \ldots, \tau_{n}\right) \sigma\left(\tau_{1}\right) \cdots \sigma\left(\tau_{n}\right)$ where $s\left(\tau_{1}, \ldots, \tau_{n}\right)$ is the order of the permutation group of the (ordered) $n$-uple $\left(\tau_{1}, \ldots, \tau_{n}\right) \in \mathcal{T}^{n}$. In the sequel we will only need to consider the subset $\mathcal{B} \mathcal{T} \subset \mathcal{T}$ which contains rooted trees with at most two sons for each vertex, this is due to the bilinear nature of the non-linear term in the NS equation.

\section{Series representation}

If we let $c_{t}(k)=|k|^{\alpha} v_{t}(k)$ the eq. (1) above takes the form

$$
c_{t}(k)=e^{-|k|^{2} t} c_{0}(k)+i \int_{0}^{t} e^{-|k|^{2}(t-s)}|k|^{\alpha} \int_{\mathbb{R}^{3}} d k^{\prime} \frac{\left\langle k, c_{s}\left(k-k^{\prime}\right)\right\rangle P_{k} c_{s}\left(k^{\prime}\right)}{\left|k-k^{\prime}\right|^{\alpha}\left|k^{\prime}\right|^{\alpha}}
$$

for function $c \in C\left(\mathbb{R}^{3} ; \mathbb{C}^{3}\right)$ such that $\sup _{t}\left\|c_{t}\right\|_{0}<\infty,\langle k, c(k)\rangle=0$ and $c(0)=0$.

For simplicity write the above equation in the abstract form

$$
c_{t}=S_{t} c_{0}+\int_{0}^{t} S_{t-s} B\left(c_{s}, c_{s}\right) d s .
$$


where $S$ is a bounded semigroup and $B$ is the symmetrized bilinear operator $B(c, d)=\left(B_{1}(c, d)+B_{1}(d, c)\right) / 2$ with

$$
B_{1}(c, d)=i|k|^{\alpha} \int_{\mathbb{R}^{3}} d k^{\prime} \frac{\left\langle k, c\left(k-k^{\prime}\right)\right\rangle P_{k} d\left(k^{\prime}\right)}{\left|k-k^{\prime}\right|^{\alpha}\left|k^{\prime}\right|^{\alpha}} .
$$

Let $\mathcal{V}=\left\{c \in C\left(\mathbb{R}^{3} ; \mathbb{C}^{3}\right): c(0)=0,\langle k, c(k)\rangle=0\right.$ and $\left.\|c\|_{0}<\infty\right\}$ and for any $T>0$ define the Banach space $\mathcal{W}_{T}=C_{b}([0, T], \mathcal{V})$ endowed with the sup norm. Define the bilinear operator $\mathcal{B}: \mathcal{W}_{T} \otimes \mathcal{W}_{T} \rightarrow \mathcal{W}_{T}$ as

$$
\mathcal{B}_{t}(c, d)=\int_{0}^{t} S_{t-s} B\left(c_{s}, d_{s}\right) d s
$$

Lemma 3.1. For any $\alpha \geq 2$ and any $T>0$, the operator $\mathcal{B}$ is well defined and there exists an increasing function $N: \mathbb{R}_{+} \rightarrow \mathbb{R}_{+}$such that

$$
\left|\mathcal{B}_{t}(c, d)\right| \leq N_{t}\|c\|_{0}\|d\|_{0}
$$

where $N_{t}$ tends to zero as $t \rightarrow 0$ for any $\alpha \geq 2$. Moreover when $\alpha=2$ we have a uniform bound $\sup _{t \geq 0} N_{t} \leq N_{*}<\infty$ independent of $T$.

PROOF. The proof can be found in the paper of Sinai [8] and consists in a direct estimate of the integral. Some general considerations on the bilinear operator are summarized in App. A.

Now define the operator $\phi: \mathcal{B T} \times \mathcal{V} \rightarrow \mathcal{W}_{T}$ by recurrence as

$$
\phi(\bullet ; h)=\mathcal{B}(S . h, S . h) \quad \phi([\tau] ; h)=2 \mathcal{B}(S . h, \phi(\tau ; h))
$$

and

$$
\phi\left(\left[\tau_{1} \tau_{2}\right] ; h\right)=2 \mathcal{B}\left(\phi\left(\tau_{1} ; h\right), \phi\left(\tau_{2} ; h\right)\right)
$$

for any $h \in \mathcal{V}, \tau, \tau_{1}, \tau_{2} \in \mathcal{B \mathcal { T }}$ where we let $(S . h)_{t}=S_{t} h$.

To find solutions of eq. (3) in $\mathcal{W}_{T}$ with initial condition $h \in \mathcal{V}$ we set up Picard iterations $\left\{u^{(n)} \in \mathcal{W}_{T}\right\}_{n}$ as $u_{t}^{(0)}=S_{t} h$ and $u_{t}^{(n+1)}=S_{t} h+\mathcal{B}_{t}\left(u^{(n)}, u^{(n)}\right)$.

LEMMA 3.2. The functions $u^{(n)}$ have the representation

$$
u^{(n)}=S . h+\sum_{\tau \in \mathcal{B} \mathcal{T}_{n-1}} \frac{1}{\sigma(\tau)} \phi(\tau ; h)
$$

where $\mathcal{B T}_{n} \subset \mathcal{B T}$ is the set of rooted trees for which the leaves are at distance at most $n$ from the root and where we conventionally let $\mathcal{B \mathcal { T }}-1=\emptyset$. 
PROOF. It is clear that the formula holds for $n=0$ (the sum does not contain any terms). Assume it holds for any $k \leq n$ and let us prove it for $n+1$ :

$$
\begin{aligned}
& u^{(n+1)}= S . h+\mathcal{B}\left(u^{(n)}, u^{(n)}\right) \\
&= S . h+\mathcal{B}(S . h, S . h)+2 \sum_{\tau \in \mathcal{B \mathcal { T }} n-1} \frac{1}{\sigma(\tau)} \mathcal{B}(S . h, \phi(\tau ; h)) \\
&+\sum_{\tau^{1}, \tau^{2} \in \mathcal{B} \mathcal{T}_{n-1}} \frac{1}{\sigma\left(\tau^{1}\right) \sigma\left(\tau^{2}\right)} \mathcal{B}\left(\phi\left(\tau^{1} ; h\right), \phi\left(\tau^{2} ; h\right)\right) \\
&= S . h+\phi(\bullet ; h)+\sum_{\tau \in \mathcal{B} \mathcal{T}_{n-1}} \frac{1}{\sigma(\tau)} \phi([\tau] ; h) \\
&+\sum_{\tau^{1}, \tau^{2} \in \mathcal{B} \mathcal{T}_{n-1}, \tau^{1} \neq \tau^{2}} \frac{1}{2 \sigma\left(\tau^{1}\right) \sigma\left(\tau^{2}\right)} \phi\left(\left[\tau^{1} \tau^{2}\right] ; h\right)+\sum_{\tau \in \mathcal{B} \mathcal{T}_{n-1}} \frac{1}{2 \sigma(\tau) \sigma(\tau)} \phi([\tau \tau] ; h) \\
&= S . h+\sum_{\tau \in \mathcal{B} \mathcal{T}_{n}} \frac{1}{\sigma(\tau)} \phi(\tau ; h) \\
& \text { since } \sigma([\tau \tau])=2 \sigma(\tau)^{2} \text { and } \sigma([\tau])=\sigma(\tau) .
\end{aligned}
$$

The norm convergence of the series

$$
u=S . h+\sum_{\tau \in \mathcal{B} \mathcal{T}} \frac{1}{\sigma(\tau)} \phi(\tau ; h)
$$

in $\mathcal{W}_{T}$ implies convergence of the Picard iterates $u^{(n)}$ to the element $u \in \mathcal{W}_{T}$ which satisfy eq. (3) in $[0, T]$ with initial condition $u_{0}=h$.

Define the following function $\theta: \mathcal{B T} \rightarrow \mathbb{R}:$

$$
\theta(\bullet)=2, \quad \theta([\tau])=1+\theta(\tau), \quad \theta\left(\left[\tau_{1} \tau_{2}\right]\right)=\theta\left(\tau^{1}\right)+\theta\left(\tau^{2}\right)
$$

and note that $h \mapsto \phi_{t s}(\tau ; h)$ is an homogeneous function of order $\theta(\tau)$. Always holds

$$
(|\tau|+1) / 2 \leq \theta(\tau) \leq|\tau|+1
$$

as easily proven by induction on $|\tau|$.

The following control of the coefficients of the series (8) is the main result of this note.

THEOREM 3.3. For any $\varepsilon \in[0,1)$ the following estimate is true

$$
\left|\phi_{t}(\tau ; h)(k)\right| \leq C_{\tau} e^{-|k|^{2} t /(|\tau|+1)} t^{|\tau| \varepsilon / 2}\|h\|_{0}^{\theta(\tau)}
$$

where the constants $C_{\tau}$ satisfy:

$$
C_{\left[\tau^{1} \tau^{2}\right]}=\frac{A}{\left|\left[\tau^{1} \tau^{2}\right]\right|^{\varepsilon / 2}} C_{\tau^{1}} C_{\tau^{2}}, \quad C_{[\tau]}=\frac{A}{|[\tau]|^{\varepsilon / 2}} C_{\tau}, \quad C_{\bullet}=A
$$

for some constant $A$ depending only on $\alpha$.

The proof of this theorem is reported in App. B.

REMARK 3.4. The constants $C_{\tau}$ can be chosen as follows:

$$
C_{\tau}=A^{|\tau|} \gamma(\tau)^{-\varepsilon / 2}
$$


Now we can prove the following result about existence and series representation of solutions of eq. (1) in the spaces $\Phi(\alpha, \alpha)$ ( $\alpha=2$ included).

COROLlaRY 3.5. The series (8) has the following properties:

a) for $\varepsilon \in(0,1)$ and fixed $\|h\|_{0}$ it converges in norm for small $t_{*}$ and solve the problem (2) in $\mathcal{W}_{t_{*}}$;

b) for $\varepsilon \in(0,1)$ and fixed $T$ it converges in norm in $\mathcal{W}_{T}$ for $\|h\|_{0}$ small enough;

c) for $\varepsilon=0$ (i.e. $\alpha=2$ ) and for $\|h\|_{0}$ small enough it converges in norm in $\mathcal{W}_{\infty}$ and define a global solution of the problem (2).

Proof. Using Thm. 3.3 and Remark 3.4 we find that there exists a constant $B$ such that

$$
\begin{aligned}
\left|u_{t}(k)-\left[S_{t} h\right](k)\right| & \leq \sum_{\tau} B^{|\tau|} \sigma(\tau)^{-1} \gamma(\tau)^{-\varepsilon / 2} e^{-|k|^{2} t /(|\tau|+1)} t^{|\tau| \varepsilon / 2}\|h\|_{0}^{\theta(\tau)} \\
& \leq \sum_{n \geq 1} Z_{n} B^{n} e^{-|k|^{2} t /(n+1)} t^{n \varepsilon / 2}\|h\|_{0}^{(n+1) / 2}\left(1 \wedge\|h\|_{0}^{(n+1) / 2}\right) .
\end{aligned}
$$

where $Z_{n}$ is the number of rooted trees in $\mathcal{B T}$ with $n$ vertices. The following recursive relations can be used to bound the $Z_{n}$ 's:

$$
Z_{1}=1, \quad Z_{n+1} \leq Z_{n}+\sum_{n_{1}+n_{2}=n} Z_{n_{1}} Z_{n_{2}}
$$

From this relation it is not difficult to prove that $Z_{n}$ grows at most exponentially, i.e. there exists a constant $D$ such that

$$
Z_{n} \leq D^{n}(n+1)^{-3 / 2} \text {. }
$$

(see for example $[\mathbf{9}]$, Sec.3).

Next, by induction we can prove that $\gamma(\tau) \geq 2^{|\tau|-1}$. This bound is optimal since it is saturated by the binary trees for which every path from the root to the leaves has the same length. Using this bound, eq. (9) and eq. (11) in eq. (10) we get

$$
\begin{aligned}
\left|u_{t}(k)\right| & \leq\left|\left[S_{t} h\right](k)\right|+\sum_{n \geq 1} Z_{n} B^{n} e^{-|k|^{2} t /(n+1)} t^{n \varepsilon / 2}\|h\|_{0}^{(n+1) / 2}\left(1 \wedge\|h\|_{0}^{(n+1) / 2}\right) \\
& \leq\|h\|_{0}+\sum_{n \geq 1}\left(D B t^{\varepsilon / 2}\right)^{n}(n+1)^{-3 / 2}\|h\|_{0}^{(n+1) / 2}\left(1 \wedge\|h\|_{0}^{(n+1) / 2}\right) .
\end{aligned}
$$

so the series (8) converges in norm whenever the geometric series

$$
\sum_{n \geq 1}\left(D B t^{\varepsilon / 2}\right)^{n}\|h\|_{0}^{(n+1) / 2}\left(1 \wedge\|h\|_{0}^{(n+1) / 2}\right)
$$

converges. This gives directly $a), b), c$ ). Indeed note that for $\varepsilon=0$ the dependence on $t$ disappear in this last series.

Now, lets come back to the original variables. It is clear that the function $v_{t}(k)=|k|^{-2} u_{t}(k)$ satisfy eq. (1) in $[0, T]$ when the series defining $u$ converges in $\mathcal{W}_{T}$. Here we are interested in the behavior of the global solutions when $\alpha=2$ :

COROLlary 3.6. In the case $\alpha=2$ and when $\|h\|_{2}$ is sufficiently small the global solution $v$ of eq. (1) with initial condition $h$ has the following two properties:

a) for fixed $k \in \mathbb{R}^{3} \backslash\{0\}, \lim _{t \rightarrow \infty}\left|v_{t}(k)\right|=0$; 
b) for fixed $t>0$, there exists two constants $C_{3}, C_{4}$ such that $\left|v_{t}(k)\right| \leq$ $C_{3} e^{-C_{4}|k| \sqrt{t}}$ as $|k| \rightarrow \infty$.

ProOF. By the same bounds performed in Cor. 3.5 we see that the function $v_{t}(k)=|k|^{-2} u_{t}(k)$ satisfy the inequality

$$
\left|v_{t}(k)\right| \leq e^{-|k|^{2} t}|h(k)|+\sum_{n \geq 1} C_{1}^{n}(n+1)^{-3 / 2} e^{-|k|^{2} t /(n+1)}\|h\|_{2}^{(n+1) / 2}, \quad k \neq 0
$$

for $\|h\|_{2}$ small enough to guarantee the convergence of the series

$$
\sum_{n \geq 1} C_{1}^{n}(n+1)^{-3 / 2}\|h\|_{2}^{(n+1) / 2}
$$

Then fixed $k \in \mathbb{R}^{3} \backslash\{0\}$ we have $\lim _{t \rightarrow \infty} e^{-|k|^{2} t /(n+1)}=0$ for each $n$ and we obtain that $\left|v_{t}(k)\right| \rightarrow 0$ as $t \rightarrow \infty$.

Next, we want to estimate the series at fixed $t$ and for $|k| \rightarrow \infty$ by Laplace method. Write

$$
\sum_{n \geq 1} C_{1}^{n}(n+1)^{-3 / 2} e^{-|k|^{2} t /(n+1)}\|h\|_{2}^{(n+1) / 2} \leq\|h\|_{2}^{1 / 2} \sum_{n \geq 1} e^{-|k|^{2} t /(n+1)+n \log \left(C_{1}\|h\|_{2}^{1 / 2}\right)}
$$

The exponent in the sum of the r.h.s has a maximum for $n \simeq|k| \sqrt{t} / \sqrt{\left|\log \left(C_{1}\|h\|_{2}^{1 / 2}\right)\right|}$ and so, when $|k| \rightarrow \infty$ we have

$$
\sum_{n \geq 1} e^{-|k|^{2} t /(n+1)+n \log \left(C_{1}\|h\|_{2}^{1 / 2}\right)} \leq C_{3} e^{-C_{4}|k| \sqrt{t}}
$$

where $C_{4}=2 / \sqrt{\left|\log \left(C_{1}\|h\|_{2}^{1 / 2}\right)\right|}$ and $C_{3}$ is some finite constant.

3.1. Remarks on particular subsets of trees. In the series (8) different classes of trees give different contributions. We define simple trees the trees with at most one branch at each vertex, i.e. of the form $[\cdots[\bullet] \cdots]$. Short trees are instead trees for which at each vertex we have two branches, each of which carries a fixed proportion $(\alpha$ or $1-\alpha$ ) of the vertices. Of course this will not be possible in general, so we allow the proportion to oscillate around $\alpha$ in the interval $[\alpha-\Delta \alpha, \alpha+\Delta \alpha]$, for some fixed $0<\Delta \alpha<\min (\alpha, 1-\alpha)$. Since rooted trees does not distinguish between branches at a vertex, we take here the convention that the branches are ordered by the number of vertices in the corresponding subtree. With this convention we can consider, without loosing generality, values of $\alpha \in(0,1 / 2)$.

We will denote $\mathcal{B T}_{0}$ the set of simple trees and $\mathcal{B} \mathcal{T}_{\alpha}$ the set of short trees corresponding to the proportion $\alpha$.

We have a first simple lemma:

Lemma 3.7. For $\tau \in \mathcal{B \mathcal { T }}_{0}$ we have $\gamma(\tau)=|\tau|$ !. For any $\alpha \in(0,1 / 2)$ there exists constants $D_{1}, D_{2}, D_{3}, D_{4}$ such that, for any $\tau \in \mathcal{B T}_{\alpha}$ we have

$$
D_{3}|\tau|^{-1} D_{4}^{|\tau|} \leq \gamma(\tau) \leq D_{1}|\tau|^{-1} D_{2}^{|\tau|} .
$$

Proof. The proof of the first claim is trivial. For the second, note that we can choose the constants $D_{1}, D_{2}, D_{3}, D_{4}$ such that the inequalities are true for all the trees $\tau \in \mathcal{B} \mathcal{T}_{\alpha}$ with $|\tau| \leq \bar{n}$ for some fixed $\bar{n}$ and moreover they satisfy

$$
\frac{D_{1} D_{2}^{-1}}{(\alpha-\Delta \alpha)\left(1-\alpha-\Delta \alpha-\bar{n}^{-1}\right)} \leq 1 \quad \text { and } \quad \frac{D_{3} D_{4}^{-1}}{(1-\alpha+\Delta \alpha)(\alpha+\Delta \alpha)} \geq 1 .
$$


Then we proceed by induction on $n \geq \bar{n}$. Assume the inequality is true for trees with $|\tau|<n$ and observe that, for $\tau \in \mathcal{B} \mathcal{T}_{\alpha},|\tau|=n$ we have $\tau=\left[\tau_{1} \tau_{2}\right]$ with

$$
(\alpha-\Delta \alpha) \leq \frac{\left|\tau_{1}\right|}{|\tau|} \leq(\alpha+\Delta \alpha)
$$

and

Then

$$
\left(1-\alpha-\Delta \alpha-|\tau|^{-1}\right) \leq \frac{\left|\tau_{2}\right|}{|\tau|} \leq\left(1-\alpha+\Delta \alpha-|\tau|^{-1}\right)
$$

$$
\begin{aligned}
\gamma(\tau) & =|\tau| \gamma\left(\tau_{1}\right) \gamma\left(\tau_{2}\right) \leq D_{1}^{2} \frac{D_{2}^{\left|\tau_{1}\right|+\left|\tau_{2}\right|}}{\left|\tau_{1}\right|\left|\tau_{2}\right|} \leq \frac{D_{1}^{2} D_{2}^{-1}}{(\alpha-\Delta \alpha)\left(1-\alpha-\Delta \alpha-|\tau|^{-1}\right)} \frac{D_{2}^{|\tau|}}{|\tau|} \\
& \leq \frac{D_{1}^{2} D_{2}^{-1}}{(\alpha-\Delta \alpha)\left(1-\alpha-\Delta \alpha-\bar{n}^{-1}\right)} \frac{D_{2}^{|\tau|}}{|\tau|} \leq D_{1} \frac{D_{2}^{|\tau|}}{|\tau|}
\end{aligned}
$$

and similarly we obtain $\gamma(\tau) \geq D_{3} D_{4}^{|\tau|}|\tau|^{-1}$, proving the claim.

This different behavior of the two classes of trees is responsible for different convergence properties of the sum (8) when restricted to simple or short trees.

Define

$$
w_{t}=\sum_{\tau \in \mathcal{B T}_{0}} \frac{1}{\sigma(\tau)} \phi_{t}(\tau ; h)
$$

then as consequence of Lemma 3.7 and Theorem 3.3 we have the following result:

Corollary 3.8. For $\varepsilon>0$, the series $w_{t}$ converges in $\mathcal{V}$ for every $t$ and every initial condition $h \in \mathcal{V}$ and

$$
\left|w_{t}(k)\right| \leq B^{\prime} \sum_{n=1}^{\infty} \frac{B^{n}}{n^{3 / 2}(n !)^{\varepsilon / 2}} e^{-|k|^{2} t /(n+1)} t^{n \varepsilon / 2}\left(1+\|h\|_{0}\right)^{n+1} .
$$

Proof. The estimates on the series are similar to those in Cor. 3.5, but now the coefficient $\gamma(\tau)=|\tau|$ ! goes to infinity fast enough to guarantee the convergence of the series for any time.

In [10], Sinai studied different classes of contributions to his series representation of NS. He calls the various contributions diagrams and then introduces short and simple diagrams which are analogous to short and simple trees (even if diagrams does not exactly corresponds to our trees). He then shows that the contribution of the simple diagrams cannot cause the divergence of the overall series. Corollary 3.8 is the analogous of this result in our setting.

For short trees the function $\gamma$ behaves exponentially with the size of the tree and this is not enough to make the series restricted to short trees converge for arbitrary time (when $\varepsilon>0$ ). A similar phenomenon is observed in $[\mathbf{1 0}]$ for short diagrams.

\section{acknowledgment}

The author wish to thanks the organizers and the lecturers of the CIME 2005 Summer school "SPDE in hydrodynamics: recent progress and prospects" for the nice environment and in particular Y. Sinai whose lectures on the mathematics of the Navier-Stokes system introduced the author to the problem studied in this note. Moreover useful comments by Y. Bakhtin and by an anonymous referee are gratefully acknowledged. 


\section{References}

[1] R. N. Bhattacharya, L. Chen, S. Dobson, R. B. Guenther, C. Orum, M. Ossiander, E. Thomann, and E. C. Waymire. Majorizing kernels and stochastic cascades with applications to incompressible Navier-Stokes equations. Trans. Amer. Math. Soc., 355(12):50035040 (electronic), 2003.

[2] Ch. Brouder. Runge-Kutta methods and renormalization. Eur. Phys. Jour. C 12 (2000), p. $521-534$.

[3] Ch. Brouder. Trees, renormalizations and differential equations. BIT Num. Math. 44 (2004), pp. 425438.

[4] J. C. Butcher, An algebraic theory of integration methods, Math. Comput., 26 (1972), pp. 79106.

[5] M. Cannone and F. Planchon. On the regularity on the bilinear term of solutions of the incompressible Navier-Stokes equations in $\mathbb{R}^{3}$. Rev. Math. Iberoamericana, 16 (2000), pp. $1-16$.

[6] A. Connes and D. Kreimer, Hopf algebras, renormalization and noncommutative geometry, Comm. Math. Phys., 199 (1998), pp. 203242.

[7] G. Gallavotti. Foundations of Fluid Dynamics. Translated from the Italian. Texts and Monographs in Physics. Springer-Verlag, Berlin, 2002.

[8] Ya. G. Sinai. On Local and Global Existence and Uniqueness of Solutions of the 3D-NavierStokes System on $\mathbb{R}^{3}$. Perspectives in Analysis, Conference in honor of L. Carlesons 75-th birthday. Benedicks, Jones and Smirnov, eds. Springer-Verlag, Berlin (to appear).

[9] Ya. G. Sinai. Power series for solutions of the 3D-Navier-Stokes system on $\mathbb{R}^{3}$. J. Stat. Phys., 121(5-6):779-803, 2005.

[10] Ya. G. Sinai. Diagrammatic Approach to the 3D-Navier-Stokes System , preprint (2005).

[11] Y. Le Jan and A.-S. Sznitman. Stochastic Cascades and 3D-Navier-Stokes Equations. PTRF 100(3), (1997), pp. 343-366.

[12] E. C. Waymire. Probability \& incompressible Navier-Stokes equations: an overview of some recent developments. Probab. Surv., 2:1-32 (electronic), 2005.

\section{Appendix A. Remarks on the spaces $\Phi(\alpha, \omega)$}

Without going in detailed proofs we would like to note some remarks about the natural functional spaces in which solutions of eq. (1) live. Following Sinai $[\mathbf{8}]$ define the space $\Phi(\alpha, \omega)(\alpha, \omega \geq 0)$ as the space of continuous functions $v: \mathbb{R}^{3} \rightarrow \mathbb{C}^{3}$ such that

$$
\|v\|_{\alpha, \omega}=\sup _{k \in \mathbb{R}^{3}} \psi(k)^{-1}|v(k)|<\infty
$$

where $\psi(k)=|k|^{-\alpha}$ for $|k| \leq 1, \psi(k)=|k|^{-\omega}$ for $|k| \geq 1$. Functions in this space can be bounded above by $|k|^{-\alpha}$ for small $k$ and by $|k|^{-\omega}$ for large $k$. Consider the the bilinear integral operator

$$
\widetilde{\mathcal{B}}(v, v)(t, k)=i \int_{0}^{t} e^{-|k|^{2}(t-s)} \int_{\mathbb{R}^{3}} d k^{\prime}\left\langle k, v_{s}\left(k-k^{\prime}\right)\right\rangle P_{k} v_{s}\left(k^{\prime}\right) d s
$$

appearing in the r.h.s of eq. (1). For this operator we have the bound

$$
|\widetilde{\mathcal{B}}(v, v)(t, k)| \leq \sup _{0 \leq s \leq t}\left\|v_{s}\right\|_{\alpha, \omega}^{2}|k|^{-1}\left(1-e^{-|k|^{2} t}\right) I(k)
$$

where $I(k)=\int_{\mathbb{R}^{3}} d k^{\prime} \psi\left(k-k^{\prime}\right) \psi(k)$. For $k \neq 0$ the integral $I(k)$ converges when $\omega>3 / 2$ and $\alpha<3$ and we prove easily that, when $|k| \leq 1$

$$
I(k) \leq C_{\alpha} \begin{cases}1 & \text { for } \alpha<3 / 2 \\ |\log | k|| & \text { for } \alpha=3 / 2 \\ |k|^{3-2 \alpha} & \text { for } 3 / 2<\alpha<3\end{cases}
$$


while when $|k|>1$

$$
I(k) \leq C_{\omega} \begin{cases}|k|^{3-2 \omega} & \text { for } 3 / 2<\omega<3 \\ |k|^{-\omega} & \text { for } \omega \geq 3\end{cases}
$$

This behavior translates in the following estimates for the r.h.s. of eq. (14). So when $|k| \leq 1$ :

$$
|k|^{-1}\left(1-e^{-|k|^{2} t}\right) I(k) \leq C_{\alpha} \begin{cases}|k|^{-\alpha} t^{(1-\alpha) / 2} & \text { for } \alpha<1 \\ |k|^{-\alpha}(1 \wedge|t|) & \text { for } 1 \leq \alpha \leq 2 \\ |k|^{-\alpha}|t|^{(\alpha-2) / 2} & \text { for } 2 \leq \alpha<3\end{cases}
$$

and when $|k| \geq 1$

$$
|k|^{-1}\left(1-e^{-|k|^{2} t}\right) I(k) \leq C_{\omega} \begin{cases}|k|^{2-2 \omega}\left(1-e^{-|k|^{2} t}\right) & \text { for } 3 / 2<\omega<2 \\ |k|^{-\omega}(1 \wedge|t|)^{(\omega-2) / 2} & \text { for } 2 \leq \omega \leq 3 \\ |k|^{-\omega}(1 \wedge|t|)^{1 / 2} & \text { for } 3 \leq \omega\end{cases}
$$

These bounds imply that $\widetilde{\mathcal{B}}$ maps $C([0, T], \Phi(\alpha, \omega))$ in itself whenever $\omega \geq 2$ for any $\alpha \in[0,3)$ and in this case the norm $N$ of $\widetilde{\mathcal{B}}$ is given by

$$
N_{T}=\sup _{t \leq T} \sup _{k}\left[\psi(k)^{-1}|k|^{-1}\left(1-e^{-|k|^{2} t}\right) I(k)\right]
$$

and become small with $T$ allowing a direct proof of existence and uniqueness of solutions to eq. (1) for small time. Moreover when $\alpha \in[1,2]$ the norm $N_{T}$ is uniformly bounded in $T$ and this implies existence and uniqueness of global solutions with small enough initial condition.

Note moreover that the same bounds are true on the torus (only wave-vectors $|k| \geq 1$ are important in this case) and that they always imply uniform control in time of the norm $N_{T}$ for any $\omega \geq 2$. In this case we have the existence and uniqueness of global solutions with small initial conditions whose decay at infinity is not worse than $|k|^{-\omega}$. Details can be found in $[8]$.

\section{Appendix B. Proofs}

\section{B.1. Theorem 3.3.}

Proof. We will prove the statement by induction on $|\tau|=n$. Let us assume that the estimate is true for any tree $\tau^{\prime}$ with $\left|\tau^{\prime}\right|<n$ and let us prove it for trees $\tau$ with $|\tau|=n$. Consider the case $\tau=\left[\tau^{1} \tau^{2}\right]$ with $\left|\tau^{1}\right|=p,\left|\tau^{2}\right|=q$ :

$$
\begin{aligned}
\left|\phi_{t}\left(\left[\tau_{1} \tau_{2}\right] ; h\right)(k)\right| \leq & 2 \int_{0}^{t} e^{-|k|^{2}(t-u)}\left|B\left(\phi_{u}\left(\tau_{1} ; h\right), \phi_{u}\left(\tau_{2} ; h\right)\right)\right| d u \\
\leq & 2 \int_{0}^{t} d u e^{-|k|^{2}(t-u)}|k|^{\alpha+1} \int_{\mathbb{R}^{3}} d k^{\prime} \frac{\left|\phi_{u}\left(\tau_{1} ; h\right)\left(k-k^{\prime}\right)\right|\left|\phi_{u}\left(\tau_{2} ; h\right)\left(k^{\prime}\right)\right|}{\left|k-k^{\prime}\right|^{\alpha}\left|k^{\prime}\right|^{\alpha}} \\
\leq & 2 C_{\tau^{1}} C_{\tau^{2}}\|h\|_{0}^{\theta(\tau)} \int_{s}^{t} d u e^{-|k|^{2}(t-u)} u^{\varepsilon / 2\left(\left|\tau^{1}\right|+\left|\tau^{2}\right|\right)}|k|^{\alpha+1} \\
& \cdot \int_{\mathbb{R}^{3}} d k^{\prime} \frac{e^{-\left|k-k^{\prime}\right|^{2} u /(p+1)-\left|k^{\prime}\right|^{2} u /(q+1)}}{\left|k-k^{\prime}\right|^{\alpha}\left|k^{\prime}\right|^{\alpha}} .
\end{aligned}
$$


The exponent in the integral has a maximum as a function of $k^{\prime}$ and

$$
\frac{\left|k-k^{\prime}\right|^{2} u}{p+1}+\frac{\left|k^{\prime}\right|^{2} u}{q+1} \geq \frac{|k|^{2} u}{p+q+2}
$$

for any $k^{\prime} \in \mathbb{R}^{3}$. So

$$
\begin{aligned}
& \left|\phi_{t}\left(\left[\tau_{1} \tau_{2}\right] ; h\right)(k)\right| \leq 2 C_{\tau^{1}} C_{\tau^{2}}\|h\|_{0}^{\theta(\tau)} \\
& \cdot e^{-|k|^{2} t /(p+q+2)} \int_{0}^{t} d u e^{-|k|^{2}(t-u)(p+q+1) /(p+q+2)} \\
& (u)^{\varepsilon / 2\left(\left|\tau^{1}\right|+\left|\tau^{2}\right|\right)}|k|^{\alpha+1} \\
& \int_{\mathbb{R}^{3}} d k^{\prime} \frac{1}{\left|k-k^{\prime}\right|^{\alpha}\left|k^{\prime}\right|^{\alpha}} \\
& \leq A^{\prime} C_{\tau^{1}} C_{\tau^{2}}\|h\|_{0}^{\theta(\tau)} e^{-|k|^{2} t /(p+q+2)} \\
& \cdot \int_{0}^{t} d u e^{-|k|^{2}(t-u)(p+q+1) /(p+q+2)}(u)^{\varepsilon / 2\left(\left|\tau^{1}\right|+\left|\tau^{2}\right|\right)}|k|^{4-\alpha}
\end{aligned}
$$

where, if $e$ is a unit vector in $\mathbb{R}^{3}$ we let

$$
A^{\prime}=2 \int_{\mathbb{R}^{3}} d k^{\prime} \frac{1}{\left|e-k^{\prime}\right|^{\alpha}\left|k^{\prime}\right|^{\alpha}}<\infty
$$

Consider the term on this last line of eq. (15):

$$
\begin{aligned}
& \int_{0}^{t} d u e^{-|k|^{2}(t-u)(p+q+1) /(p+q+2)}(u)^{\varepsilon / 2\left(\left|\tau^{1}\right|+\left|\tau^{2}\right|\right)}|k|^{4-\alpha} \\
& \quad=t^{\left(\left|\tau^{1}\right|+\left|\tau^{2}\right|+1\right) \varepsilon / 2} \int_{0}^{1}|\tilde{k}|^{4-\alpha} e^{-|\tilde{k}|^{2}(1-u)(p+q+1) /(p+q+2)} u^{\varepsilon / 2\left(\left|\tau^{1}\right|+\left|\tau^{2}\right|\right)} d u
\end{aligned}
$$

with $\tilde{k}=(t-s)^{1 / 2} k$. Let $a=(p+q+1) /(p+q+2)$. We have the following bound, proved in lemma B.1 below:

$$
\int_{0}^{1} e^{-|\tilde{k}|^{2}(1-u) a} u^{(p+q) \varepsilon / 2} d u \leq\left(a|\tilde{k}|^{2}+(p+q) \varepsilon / 2\right)^{-1}
$$

Gathering all together we get

$$
\left|\phi_{t}\left(\left[\tau_{1} \tau_{2}\right] ; h\right)(k)\right| \leq A^{\prime} C_{\tau^{1}} C_{\tau^{2}}\|h\|_{0}^{\theta(\tau)} e^{-|\tilde{k}|^{2} /(|\tau|+1)} t^{|\tau| \varepsilon / 2} \frac{|\tilde{k}|^{2-\varepsilon}}{a|\tilde{k}|^{2}+(p+q) \varepsilon / 2}
$$

When $\varepsilon \in(0,1)$ we have

$$
\sup _{\tilde{k} \in \mathbb{R}^{3}} \frac{|\tilde{k}|^{2-\varepsilon}}{a|\tilde{k}|^{2}+(p+q) \varepsilon / 2} \leq K[(p+q)]^{-\varepsilon / 2}
$$

where $K$ is a constant not depending on $\varepsilon$, so in this case

$$
\left|\phi_{t}\left(\left[\tau_{1} \tau_{2}\right] ; h\right)(k)\right| \leq A^{\prime \prime} C_{\tau^{1}} C_{\tau^{2}}\|h\|_{0}^{\theta(\tau)} e^{-|\tilde{k}|^{2} /(|\tau|+1)} \frac{t^{|\tau| \varepsilon / 2}}{|\tau|^{\varepsilon / 2}}
$$

When $\varepsilon=0$ we have instead the bound

$$
\left|\phi_{t}\left(\left[\tau_{1} \tau_{2}\right] ; h\right)(k)\right| \leq A^{\prime \prime} C_{\tau^{1}} C_{\tau^{2}}\|h\|_{0}^{\theta(\tau)} e^{-|\tilde{k}|^{2} /(|\tau|+1)}
$$

Proving the claim in this case. The other cases can be treated similarly. 
LEMMA B.1.

$$
\int_{0}^{1} e^{-a(1-u)} u^{b} d u \leq 1 \wedge(a+b)^{-1}
$$

Proof. Easy:

$$
\begin{aligned}
\int_{0}^{1} e^{-a(1-u)} u^{b} d u & =\int_{0}^{1} e^{-a u}(1-u)^{b} d u \int_{0}^{1} e^{-a u-b u} d u \\
& \leq \int_{0}^{\infty} e^{-(a+b) u} d u=(a+b)^{-1}
\end{aligned}
$$

Dip. Di Matematica Applicata "U. Dini" - Università di Pisa, Italia E-mail address: m.gubinelli@dma.unipi.it 